\title{
Collaborative Wireless Power Transfer in Wireless Rechargeable Sensor Networks
}

\author{
Azka Amin, ${ }^{1}$ Xi-Hua Liu $\mathbb{D}^{1},{ }^{1}$ Muhammad Asim Saleem $\mathbb{D}^{,}{ }^{2}$ Shagufta Henna, ${ }^{3}$ \\ Taseer-ul Islam, ${ }^{4}$ Imran Khan, ${ }^{5}$ Peerapong Uthansakul ${ }^{1},{ }^{6}$ Muhammad Zeshan Qurashi, \\ Seyed Sajad Mirjavadi, ${ }^{7}$ and Masoud Forsat ${ }^{7}$ \\ ${ }^{1}$ School of Business, Qingdao University, Qingdao 266061, China \\ ${ }^{2}$ School of Information and Software Engineering, University of Electronic Science and Technology, Chengdu 610054, China \\ ${ }^{3}$ Department of Computing, Letterkenny Institute of Technology, Co. Donegal, Ireland \\ ${ }^{4}$ Bahria University, Islamabad, Pakistan \\ ${ }^{5}$ Department of Electrical Engineering, University of Engineering and Technology, Peshawar, Pakistan \\ ${ }^{6}$ School of Telecommunication Engineering, Suranaree University of Technology, Thailand \\ ${ }^{7}$ Department of Mechanical and Industrial Engineering, College of Engineering, Qatar University, P.O. Box 2713 Doha, Qatar
}

Correspondence should be addressed to Xi-Hua Liu; xihua-liu@163.com and Peerapong Uthansakul; uthansakul@sut.ac.th

Received 4 January 2020; Revised 8 April 2020; Accepted 14 May 2020; Published 30 June 2020

Academic Editor: Manuel Fernandez-Veiga

Copyright (C) 2020 Azka Amin et al. This is an open access article distributed under the Creative Commons Attribution License, which permits unrestricted use, distribution, and reproduction in any medium, provided the original work is properly cited. The publication of this article was funded by Qatar National Library.

\begin{abstract}
Wireless power transfer techniques to transfer energy have been widely adopted by wireless rechargeable sensor networks (WRSNs). These techniques are aimed at increasing network lifetime by transferring power to end devices. Under these wireless techniques, the incurred charging latency to replenish the sensor nodes is considered as one of the major issues in wireless sensor networks (WSNs). Existing recharging schemes rely on rigid recharging schedules to recharge a WSN deployment using a single global charger. Although these schemes charge devices, they are not on-demand and incur higher charging latency affecting the lifetime of a WSN. This paper proposes a collaborative recharging technique to offload recharging workload to local chargers. Experiment results reveal that the proposed scheme maximizes average network lifetime and has better average charging throughput and charging latency compared to a global charger-based recharging.
\end{abstract}

\section{Introduction}

A WSN is a network of hundreds of sensor devices deployed randomly in an area. Under most deployments, these sensor nodes are autonomous and are small. In a typical sensor network deployment, a base station (BS) is responsible to process data after collecting it from sensor nodes. These deployed sensor nodes forward data to the sink node either based on a single event, forwarding this information to the sink or next relay node, and processing and aggregation. These functions characterize WSN as an autonomous selforganized network. Apart from simple operations as discussed above, some WSN applications may require complex computations which make it a highly energy-driven network requiring immediate energy replenishment for its continuous operation [1]. This energy-driven nature has attracted significant attention for the last decade and still is considered as one of the challenging issues. Battery replacement is challenging and difficult in certain circumstances, e.g., hazardous areas where deployed sensors are difficult to access. Network lifetime can be prolonged for continuous operations with the help of energy-efficient scheduling, charging, and harvesting mechanisms [1]. Most wireless energy transfer techniques have revolutionized the way to recharge sensor devices and are widely adopted by WRSNs. In these techniques, BS also called coordinator access point or sink node emits radio frequency (RF) waves to recharge sensor nodes. Kurs et al. [2] effectively transferred energy wirelessly from one magnetic 
object to another. Inventors powered the light bulb of $60 \mathrm{~W}$ efficiency from two meters. Further research carried by the industry achieved $75 \%$ of efficiency for transferring a $60 \mathrm{~W}$ power wirelessly from three meters. This research resulted in the development of several commercially used products such as cell phones, RFIDs, sensors, and automotive vehicles which are based on the principles of wireless power transfer (WPT).

In recent times, few energy harvesting techniques have been proposed to replenish a sensor's battery to prolong the network lifetime. These schemes, however, require installation of external instruments like wind turbines and solar panels and are helpful to improve network lifetime under certain conditions. It is not feasible to completely rely on these techniques as they cannot work in the absence of energy sources. More reliable approaches to replenish a node's battery must be investigated [1]. The major hurdle to the successful operation of these power transfer techniques are the limitations imposed by the propagation nature of waves. The strength of the signal decreases as signals propagate over longer distances limiting the efficient operation of low-power devices. Few recharging schemes operate ondemand where a node may request recharging only when it is about to deplete all its energy. If its charging demand is not fulfilled in time, it may die out, thereby affecting the network lifetime and connectivity which is critical for emergency applications. A multisink scenario with varying charging demands may result in this situation. In this situation, when a sink node places a charging request to a BS, the BS may be already busy transferring the power to another sink. In that case, the request of the sink node cannot be fulfilled on time and needs to be queued. This delay can result in the requesting sink node to deplete all its energy and die which affects the overall network life. This work proposes a novel solution to address this problem with the help of local charger deployments in different regions of a WSN which can work collaboratively to serve the charging request from their local region in a timely fashion. Specifically, this work investigates the limitations and challenges of different WPT techniques and proposes a novel WPT technique called zoning and collaborative charging scheduling (ZCCS) scheme to improve the charging time and charging throughput in critical regions. The main contributions of the paper are summarized as follows:

(i) The paper proposes a charger deployment model to accelerate the recharging of sensor nodes deployed in different regions

(ii) Based on the charger deployment model, we also propose an on-demand recharging protocol called as ZCCS

(iii) The work performs various simulations to establish the better charging percentage and charging latency of ZCCS against NaZCCS (global charger-based) under various network densities

The rest of the paper is organized as follows. Related work is reviewed in Section 2. The network model and prob- lem description are described in Section 3. The proposed algorithm called ZCCS is elaborated in Section 4. In Section 5 , experimental setup, performance metrics, results, and analysis for both ZCCS and NaZCCS are presented. Finally, Section 6 concludes the paper.

\section{Related Work}

Several models have been proposed for wireless power transfer with various objectives. Dai et al. [3] explore safe charging problem under a sensor network deployment with a static sink. The proposed work keeps the unexpected omission of electromagnetic radiated waves from multiple sinks below a predefined threshold. Selection of an ideal point for electromagnetic radiated signals in a plane is one of the challenges which need consideration. The authors in [3] propose a near optimal solution to address this problem. Rechargeable sensors have potential to harvest energy from other sources and are capable to maximize the charging efficiency. The authors in [4] discussed the problem to place chargers in a wireless sensor network. Although the proposed approach is time efficient, it requires a higher number of chargers to fulfill the charging demand of the area. The authors in [5] determine an optimal reader power towards balanced energy charging to avoid transmission collision. The proposed scheme ensures that the total duration for all wireless identification and sensing platforms (WISPs) to be fully charged is minimized. However, as the number of WISPs increases, the power of reader decreases.

A work on joint data gathering and energy replenishment has been reported in $[6,7]$. In [6], Zhang et al. focused on the maximum utility of the network and proposed an algorithm to improve routing, sensing, and energy management. The authors also proposed an energy management scheme in the dynamic environment along with a topology control scheme to reduce computational complexity. It also improved data gathering efficiency; however, the proposed scheme experiences security loopholes. The work proposed by Zhang et al. [6] is aimed at maximizing the utility of users in a network. The proposed technique is highly efficient and computationally less complex. This solution can be approximated by a distributed algorithm using standard optimization techniques. It achieved better performance and has a smaller battery size requirement with low complexity online solution. However, the proposed solution does not consider wireless interference and requires complex statistical information for its operation. FarMAC [8] uses more various power sources to maximize energy efficiency.

It achieves this with the help of concurrent data gathering and energy replenishment. Although FarMAC improves overall network throughput, it incurs higher delay in data collection. This delay is more significant when the number of sensor nodes deployed in an area is approximately five times greater than the number of sinks.

Propagation limitations of energy signals result in power decreases with an increase in distance. Khan et al. [9] proposed a zoning technique to address the propagation limitations of waves. In this work, the network area is distributed into different zones and sectors. The authors proposed a 
protocol for an efficient medium access which extends time periods to increase the lifetime of waves. However, the proposed scheme is subject to bandwidth limitations. Wireless power transfer in WSN deployments with mobile chargers for network optimization has been investigated in [10-17].

In [10], Xie et al. presented a cellular structure in which they partition the area into hexagonal-shaped cells. The authors proposed an enhanced framework to improve recharging time and path determination. The proposed scheme also addressed the selection of the charging location for optimal power transfer. However, the proposed scheme does not consider the number of charged sensor nodes under high traffic loads.

Cooperation of mobile chargers (MCs) to optimize charging is discussed in [10-12]. These techniques allow MCs to harvest energy from each other when required. The authors in [11] proposed scheduling algorithms to improve the overall energy usage effectiveness of a WSN. Although the proposed approach improves charging coverage, it does not consider the issue of charging reliability under dense sensor deployments. In [10], the authors analyse the relationship between traveling cost and transferred energy in a wireless rechargeable sensor network with mobile chargers. The proposed solution demonstrated network lifetime improvement in different scenarios. Collaboration between the MCs has potential to improve charging efficiency. In [12], MCs are divided into two categories; one of them can charge sensors called mobile chargers, whereas the second can charge mobile chargers called special chargers. The proposed cooperative charging scheme shows better charging capability with improved coverage and quality. It also significantly improves network lifetime with guaranteed connectivity in the network. The proposed has potential to be merged with noncollaborative protocols for improved performance. In this technique, only sink node performs data gathering process with no burden on sensor nodes. The proposed protocol is not feasible for nonuniform network deployments.

Xie et al. [13] studied the colocation problem of WPTenabled charger and base station for data collection on a vehicle called as wireless charging vehicle (WCV). In [14], Zhang et al. proposed a scheme to maximize network usage through efficient data collection. It is aimed at collecting maximum data while maintaining the network fairness. However, the proposed scheme experiences a trade-off of fairness and network throughput. In [15], the authors present a scheduling algorithm to maximize the network lifetime through energy replenishment. The proposed scheduling approach has certain practical limitations to get the exact location of nodes. An algorithm proposed in [16] is aimed at conserving energy with a minimum number of chargers. However, this scheme incurs high overhead.

An increase in distance and variation in angle may impact the efficiency of a charger. These considerations are addressed by Rao et al. in [17] where the authors proposed a scheme to minimize charging and travelling time for a mobile charging rechargeable sensor deployment. The proposed scheme performs well in terms of charging time; however, the adjustment of angle and distance is challenging under varying speeds. The joint operation of WPT-enabled charger and base station on the same vehicle has been considered by the works in [10-17]. However, efficient operation of this mechanism is challenging in hazardous areas where deployed sensors are difficult to access. In a recent work [18], a deterministic method to construct a recharging path for mobile chargers to charge sensors has been proposed. The method, however, requires track of residual energy, location, and energy consumption rate. The authors in [19] proposed a recharging schedule by considering multisensory model to charge multiple sensors simultaneously with improved charging efficiency. The sdm recharging schedule relies on the velocity control coupled with spatial and temporal discretization.

In a work by Liu et al. [16], an energy-efficient recharging solution to address the minimum charger recharging problem has been proposed. In [20], Lin et al. proposed a charging scheme based on double thresholds of residual energy. To accelerate the recharging process, two charging schemes called hierarchical clustering charging algorithm and hierarchical clustering charging algorithm based on task splitting are proposed in [21]. In [22], a temporal-spatial real-time charging scheduling algorithm to maximize the network lifetime by reducing the number of dead nodes has been introduced. The authors in [23] proposed a recharging schedule algorithm called TADP to recharge sensor nodes according to temporal and distance priorities. The authors in [24] proposed a recharging scheduling algorithm to recharge the sensor nodes by using a charging vehicle. The scheduling algorithm considers the traveling distance of charging a vehicle to recharge the sensor nodes to maximize the network lifetime. In [10], the authors formulate the recharging problem as a traveling salesman problem with two key considerations including energy replenishment and the vehicle traveling cost.

According to the authors in [25], charging latency is one of the primary concerns in wireless rechargeable sensor networks. To improve this, the authors proposed a solution to group and recharge sensors according to their energy consumption rate. The solution, however, does not focus on the average charging throughput of the network which can contribute significantly to the network maximization. In another work in [15], the authors considered the network lifetime maximization as the coverage problem. They solved the problem by devising a charging schedule based on linear programming. Some works consider the chargers with the limited power supply available to charge sensor networks. In a work in [26], Ma et al. determined an energy-efficient charging path based on the residual energy of sensor nodes.

In [27], the authors have proposed a particle swarm optimization- (PSO-) based clustering approach to replenish the energy of sensor nodes. The proposed approach exploits wireless power transfer technique coupled with dynamic recharging schedule. The proposed scheme has demonstrated significant improvement in charging efficiency compared to grid clustering. The work, however, relies on a charging vehicle to achieve the objective. In another study [28], the authors have proposed an energy-efficient algorithm for wireless charging vehicles. This work differs from the work in [27], in that wireless power transfer is enabled for a 
multihop scenario. The approach uses adaptive transfer function to select a cluster head for each region. Specifically, the cluster head selection is based on the residual energy and position of sensors within a cluster.

The authors in [29] have proposed a reinforcement learning-based recharging algorithm. The proposed algorithm recharges sensor nodes by using a fixed charger for less critical regions; however, critical sensor nodes are recharged through a mobile charger. The algorithm exploits reinforcement learning to devise an optimal policy for the charging path. The results of the proposed scheme have maximized the network lifetime compared to other state-of-the-art recharging algorithms. The trade-off between the exploration and exploitation makes this solution expensive compared to other heuristics.

Most of the above work addresses the network lifetime maximization problem based on the energy consumption rate and coverage or through the determination of a charging path determination. However, none of the above-discussed schemes propose a solution to maximize the network lifetime in an on-demand manner for the dense deployment in a region. Compared to the above work on recharging wireless sensor networks, this work proposes an on-demand network maximization which is aimed at maximizing the lifetime of a denser charger deployment without relying on in-hand scheduling. The proposed scheme offloads the charging workload from the global chargers to local chargers, thus improving the average charging throughput of the network.

\section{Problem Description and Network Model}

3.1. Problem Description. In wireless rechargeable networks, energy consumption rate of each sensor node varies due to variations in an environment including location and density of the network [30]. This work proposes an on-demand collaborative charging technique to address the following problems:

(i) Consideration to on-demand energy charging compared to the scheduled charging in traditional wireless rechargeable sensor networks

(ii) Charging multiple sensors simultaneously deployed in different regions

(iii) To replenish energy of a network with minimum latency by distributing recharging load across local chargers

3.2. Network Model. The proposed technique assumes two types of chargers deployed in the area called as global charger (GC) and local chargers (LCs). GC is deployed in the centre of the network to ensure that all LCs are in its transmission range. Each region is equipped with a LC which can charge its sensor nodes. The LC is assumed to collect and aggregate data from the sensors and forward to the GC which may incur extra energy. The LC is assumed to be equipped with significantly higher energy compared to the sensor nodes. This deployment is illustrated in Figure 1. In each region, the deployment of sensor nodes is assumed random, static,

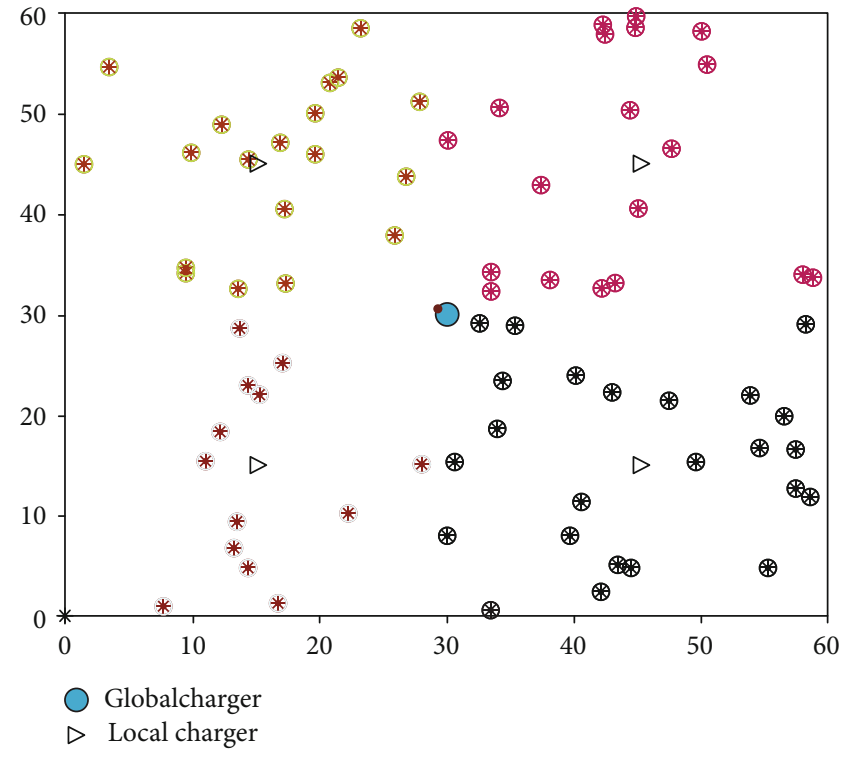

Figure 1: Network model for the ZCCS (area in meters).

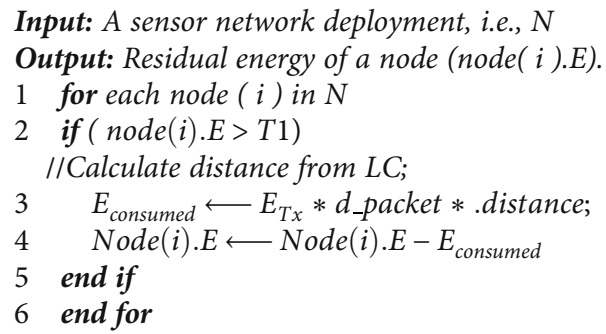

Algorithm 1: Communication.

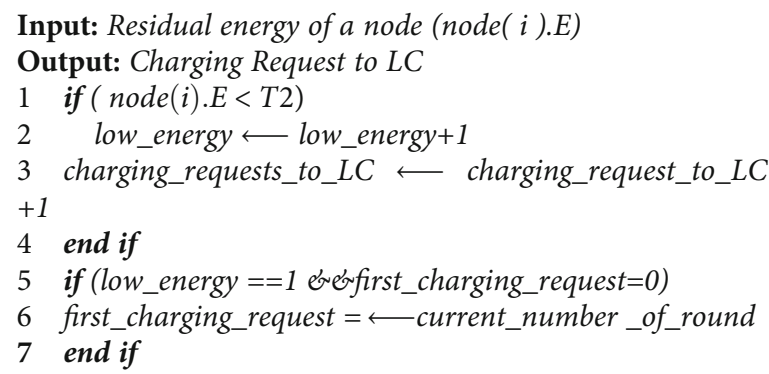

Algorithm 2: Recharging requirement for nodes.

and uniform. All sensor nodes are assumed to be equipped with homogeneous capabilities with respect to the antenna gain. Both the sensor nodes and LCs have maximum battery capacity denoted as Et. These devices are equipped with a transceiver to harvest energy from the LC and GC.

Our experiments divide the simulation area into 4 regions each of size $15 \times 15 \mathrm{~m}^{2}$ as shown in Figure 1. Nodes are randomly deployed in each region. Charging range of each LC is $15 \mathrm{~m}$ to cover its region with the assumption that 


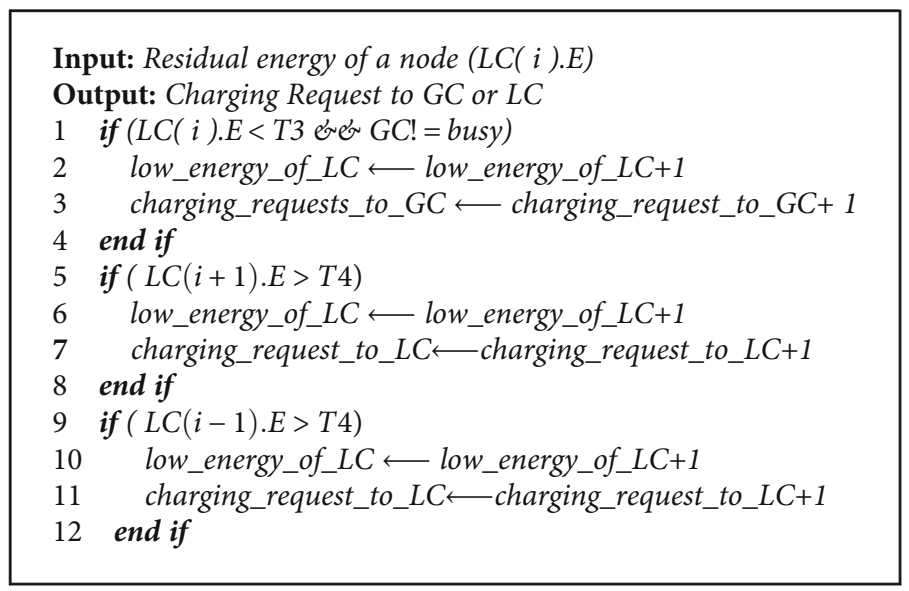

Algorithm 3: Recharging requirement for LCs.

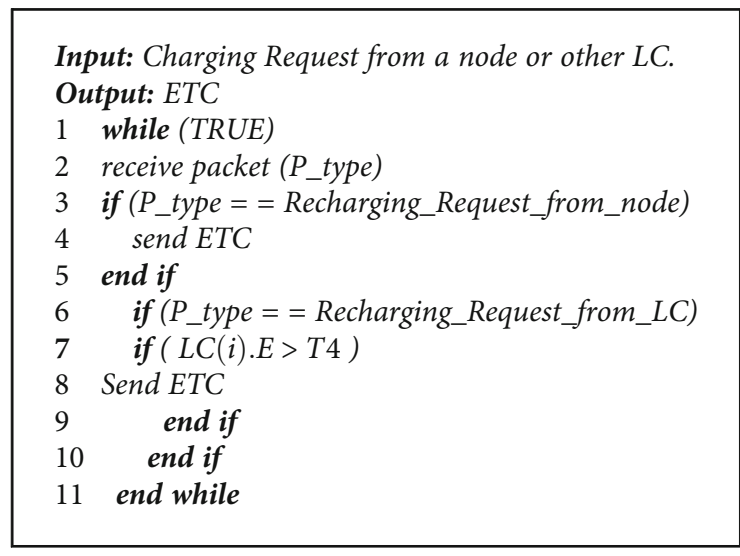

Algorithm 4: Recharging confirmation.

each LC can recharge sensors in its local region. If energy experienced by a LC drops below the threshold, the LC initiates an on-demand recharging request from the GC based on Algorithm 4 as discussed in Section 4.3. Each LC is assumed to be deployed in the centre of the region; therefore, the transmission range of GC is 15 to make sure that all the LCs are recharged as illustrated in Figure 1.

Both the sensor nodes and LCs initiate a charging request when their energy drops lower than a predefined threshold. The charging request carries a node's ID and its remaining energy. LC and GC buffer incoming charging requests and serve them in the order of their residual energy. It is also assumed that the dimensions of the sensor network are known along with the coordinates of the GC and LCs. Messages used in the proposed WPT technique are described briefly in Table 1 .

\section{Proposed WPT technique}

This section introduces the operation of zoning and collaborative charging scheduling scheme to address the problems discussed in Section 3.1. The scheme consists of different phases including communication, recharging request, recharging confirmation, and wireless power transfer. These phases are illustrated below in detail.
Table 1: Protocol messages.

\begin{tabular}{lc}
\hline Terminologies & Description \\
\hline Node $(i) . E$ & Current energy of node $i$ \\
$E_{\text {consumed }}$ & Consumed energy of a node \\
Node ID & Identity of a node \\
Distance & Distance of a node from its LC \\
ETC & Energy transfer confirmation \\
\hline
\end{tabular}

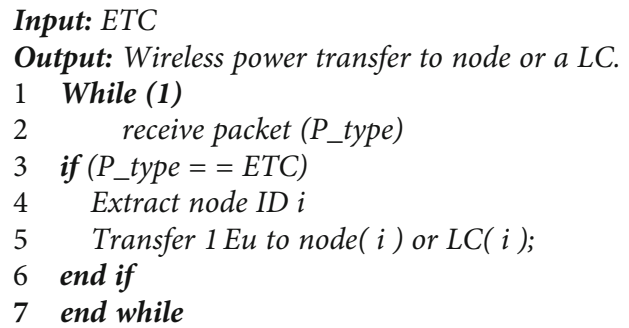

Algorithm 5: Wireless power transfer.

4.1. Phase I: Communication. Each sensor node keeps track of its residual energy to initiate wireless power transfer. Residual energy of each sensor node depends on different functions including sensing, forwarding, and aggregation. These functionalities result in different energy consumption rates at different sensor nodes. Once the residual energy of these sensors drops below threshold $\mathrm{T} 1=0.1 \mathrm{~J}$, it initiates a charging request to the LC. As the distance of the sensor nodes is different from the LC, charging requests may arrive in different orders at the LC even if initiated at the same time. A LC always serves the request in the order of the residual energy. This is illustrated in Algorithm 1 which runs in a decentralized manner on each sensor node. Energy consumed by a sensor node depends on its distance from the sender or receiver. In the algorithm, in lines 2 to 4 , each sensor node keeps track of its residual energy and as it drops below the threshold T1, sensor node enters in Phase II. 


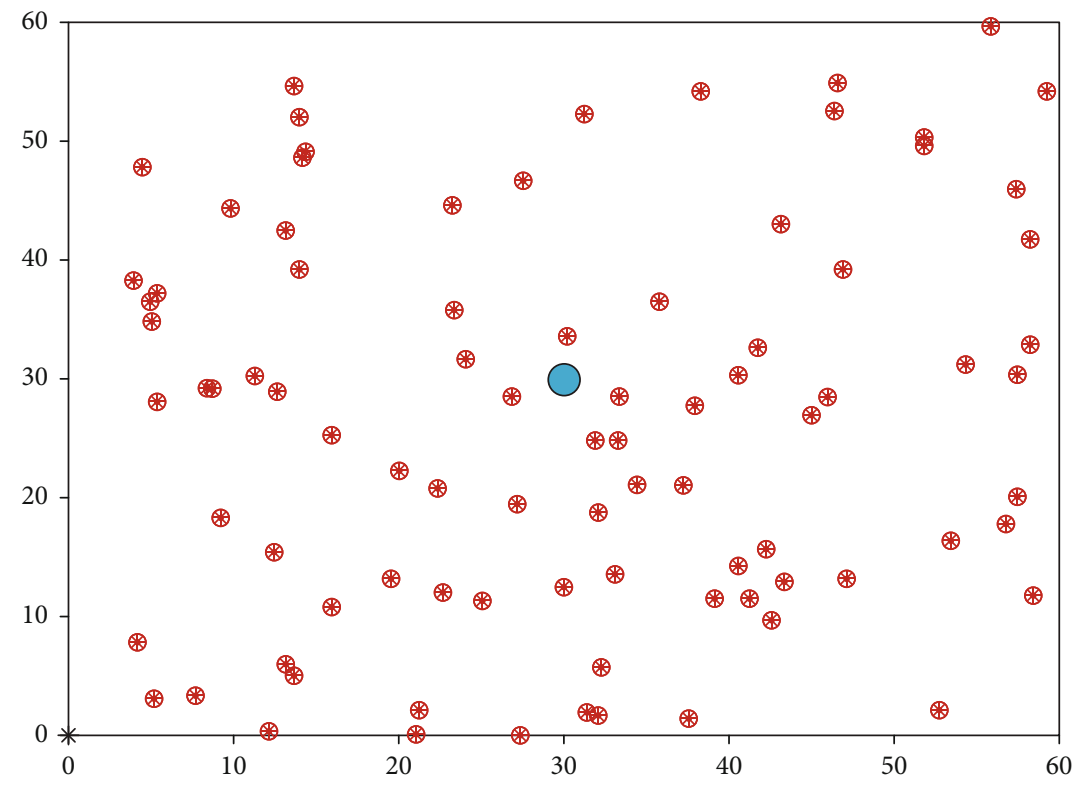

FIgURE 2: Network model for NaZCCS (area in meters).

4.2. Phase II: Recharging Request. If a sensor node experiences low-energy issue, i.e., its node $(i)$.E is below the predefined threshold, i.e., $\mathrm{T} 2=0.4 \mathrm{~J}$, it sends a recharging request to the LC. This charging request consists of node ID and its current energy status. The LC records the charging request and extracts the node ID and the node energy. The number of recorded charging requests is used later to evaluate the charging throughput as discussed in Section 5. The LC schedules the recharging of received requests in the ascending order of node( $i$ ).E, where $i$ denotes the sensor node ID. The recharging request procedure for the sensor nodes is illustrated in Algorithm 2.

Similarly, when the energy of a LC denoted as LC(i).E drops below the predefined threshold, i.e., T3 $=2 \mathrm{~J}$, it sends a recharging request to the $\mathrm{GC}$ in a similar manner as described above. This request contains local charger ID and its current energy. Upon receiving multiple recharging requests, the GC schedules recharging of LCs in the ascending order of $\mathrm{LC}(i)$.E, where $i$ denotes the local charger ID. If the GC is busy charging another LC, the requesting LC initiates the recharging request procedure with its neighbouring LCs. This is illustrated in Algorithm 3.

4.3. Phase III: Recharging Confirmation. Upon receiving a recharging request from a sensor node, the LC evaluates request based on the number of requests in the queue and its available energy. If a recharging request is received from another local charger, depending on the available energy and number of pending recharging requests, the LC decides to fulfill the recharging request. It queues the request if it can fulfill the request within a specified time. It can be observed from Algorithm 4 that the LC confirms a recharging request only if its energy, i.e., LC.E is greater than the predefined threshold defined as T4 $=4 \mathrm{~J}$. Lines 2 to 5 of Algorithm 4 show that a recharging confirmation is sent to a sensor node after receiving its recharging request. On the other hand, lines 6 to 9 illustrate that the LC sends the recharging confirmation to its neighbouring local charger on receiving their request and after evaluating the available energy.

LCs sort the service pool from small to large energy and schedule the recharging requests with less remaining energy. In other words, the actual charging sequence is determined based on the remaining lifetime of sensor nodes. The process to charge the next sensor node or LC is on-demand and dynamic and is adjusted each time a new recharging request is received by the $\mathrm{LC}$.

4.4. Phase IV: Wireless Power Transfer. Once a LC or sensor node receives a recharging confirmation from the $\mathrm{LC}$ or $\mathrm{GC}$, it keeps itself ready to receive the power/energy which is transferred in the form of energy unit (Eu). If recharging request has been fulfilled, a sensor node and LC evaluate their remaining energy. LC keeps track of the number of fulfilled recharging request to compute the charging throughput and charging percentage. Steps for the wireless power transfer from an LC to another LC or sensor node are illustrated in Algorithm 5. Line 2 of Algorithm 5 shows that if a LC receives a ready to recharge packet from a sensor or $\mathrm{LC}$ it transfers $1 \mathrm{Eu}$ to the $\mathrm{LC}$ or sensor node until the requested energy is transferred as given in lines 4 to 5 .

\section{Experimental Setup}

This section evaluates the performance of ZCCS and compares it with the naive version of ZCCS, called as NaZCCS. In NaZCCS, all the sensor nodes deployed in the region are charged by a single global charger located in the centre of the region. Performance metrics used for the evaluations are described in Section 5.1, and performance evaluations under various scenarios for the experiments are given in Section 5.2. 


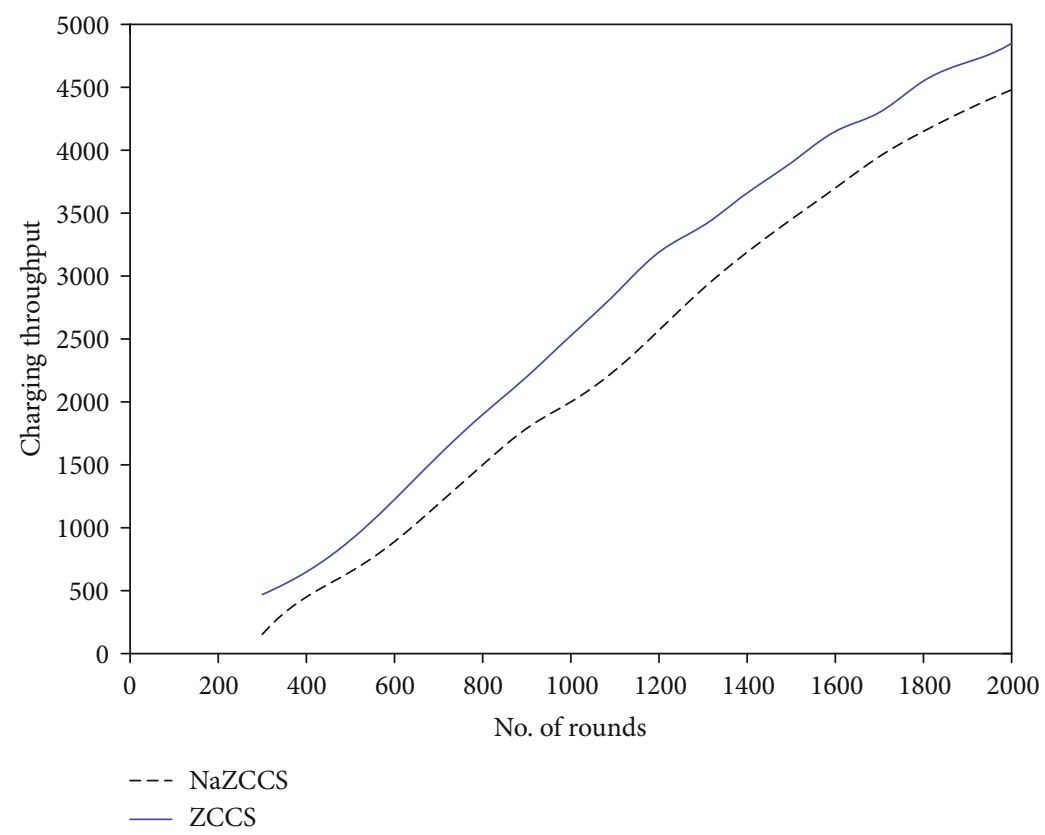

Figure 3: Average charging/round under node density 0.1.

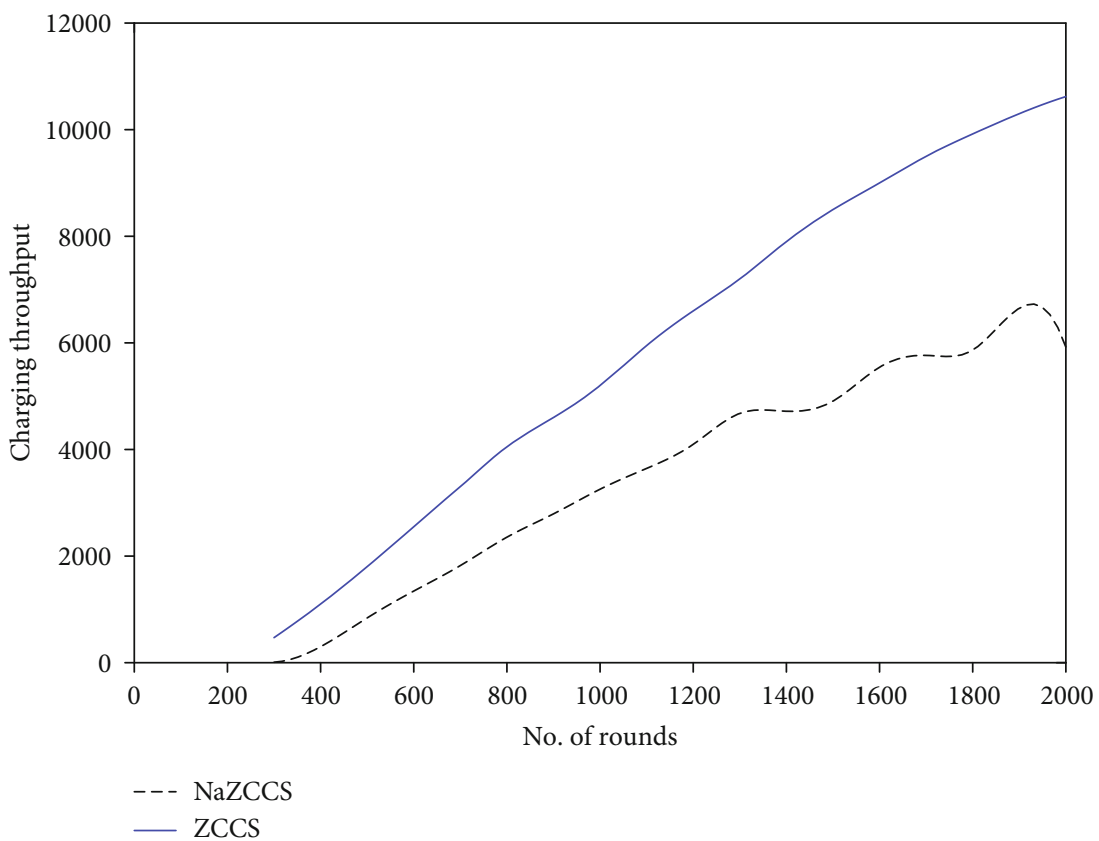

FIgURe 4: Average charging/round at node density 0.2 .

5.1. Performance Metrics. To evaluate the performance of ZCCS and compare it with the NaZCCS as shown in Figure 2, this paper introduces a notion of charging throughput. Charging throughput represents the number of sensor nodes charged per unit time. ZCCS strives to maximize the charging throughput to ensure maximum network lifetime. It assures that a minimum number of sensor nodes drain out energy and are recharged in less time. In other words, charging throughput indirectly represents the average functioning time of sensor nodes.
The dynamic nature of sensor networks makes it hard to predict a sensor's functioning time; therefore, performance evaluations consider it as the total number of sensors charged in unit time.

Compared to the existing recharging scheduling techniques, NaZCCS operates in an on-demand manner and requires an exchange of different control packets which are exchanged between the sensor and local charger and global chargers. Exchange of these control chargers may incur extra energy. 


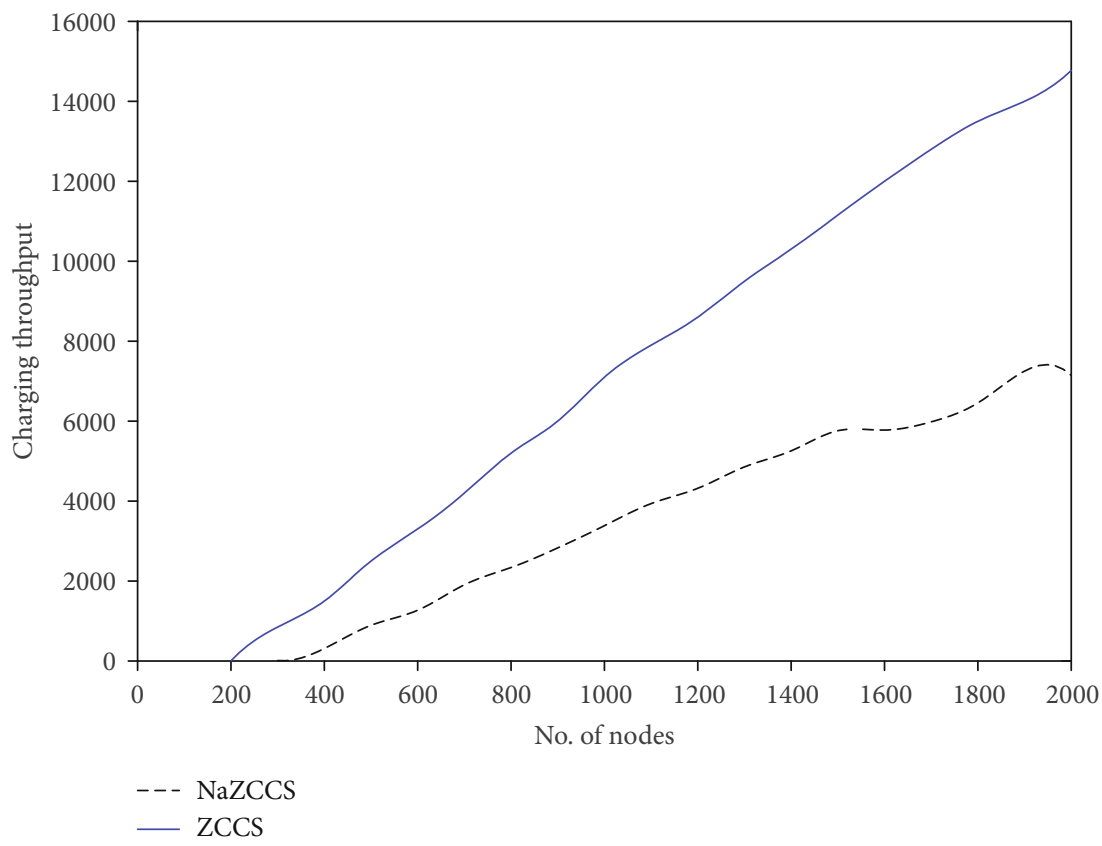

FIGURE 5: Average charging/round under node density 0.3.

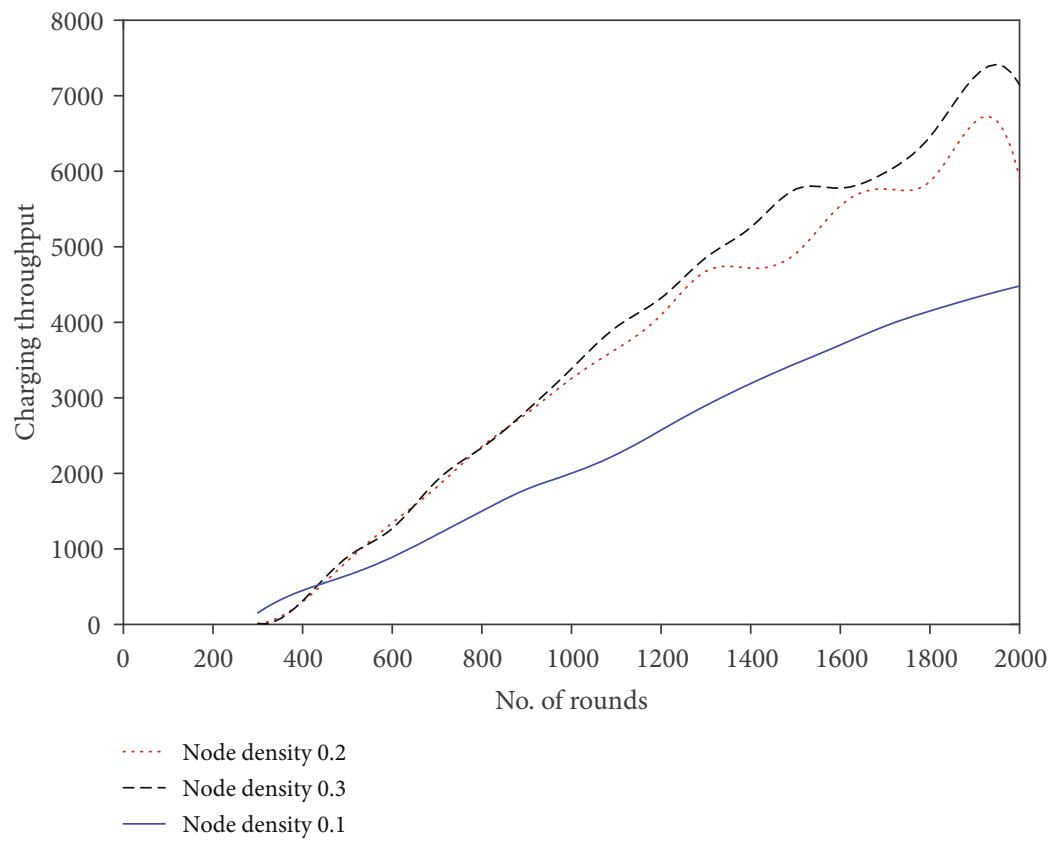

FIgURE 6: Average charging throughput of NaZCCS in each round under different node densities.

In our evaluations, energy overhead represents the number of control packets exchanged among the sensors and chargers to fulfill a charging request of 1 Eu. Like WPT, through the magnetic resonant coupling, an LC must send $100 \mu \mathrm{J}$ pulses towards a sensor node to charge one energy unit $(1 \mathrm{Eu}=50 \mu \mathrm{J})$. Results are averaged over 5 runs. To evaluate how faster ZCCS can replenish the energy of sensor nodes, we also consider the average charging latency experienced by the sensor nodes. Charging latency of a sensor node is defined as the time when the sensor node sends the recharging request to the time the local charger starts recharging the sensor node.

\subsection{Performance Evaluations}

5.2.1. Charging Throughput under Varying Node Densities and Rounds. This section evaluates the charging throughput of both the ZCCS and NaZCCS under varying node densities and rounds. Node density is defined as the number of sensor nodes deployed per unit area. The experiments under 


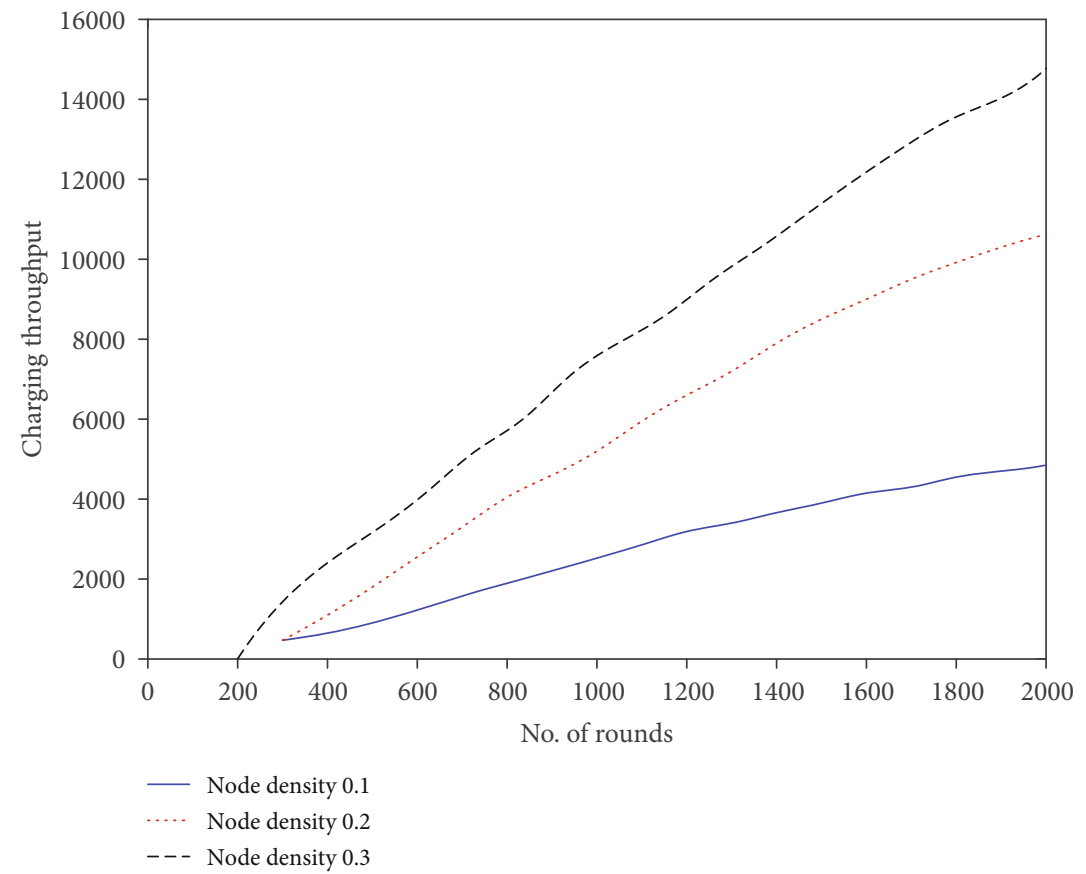

FIGURE 7: Average charging throughput of ZCCS in each round under different node densities.

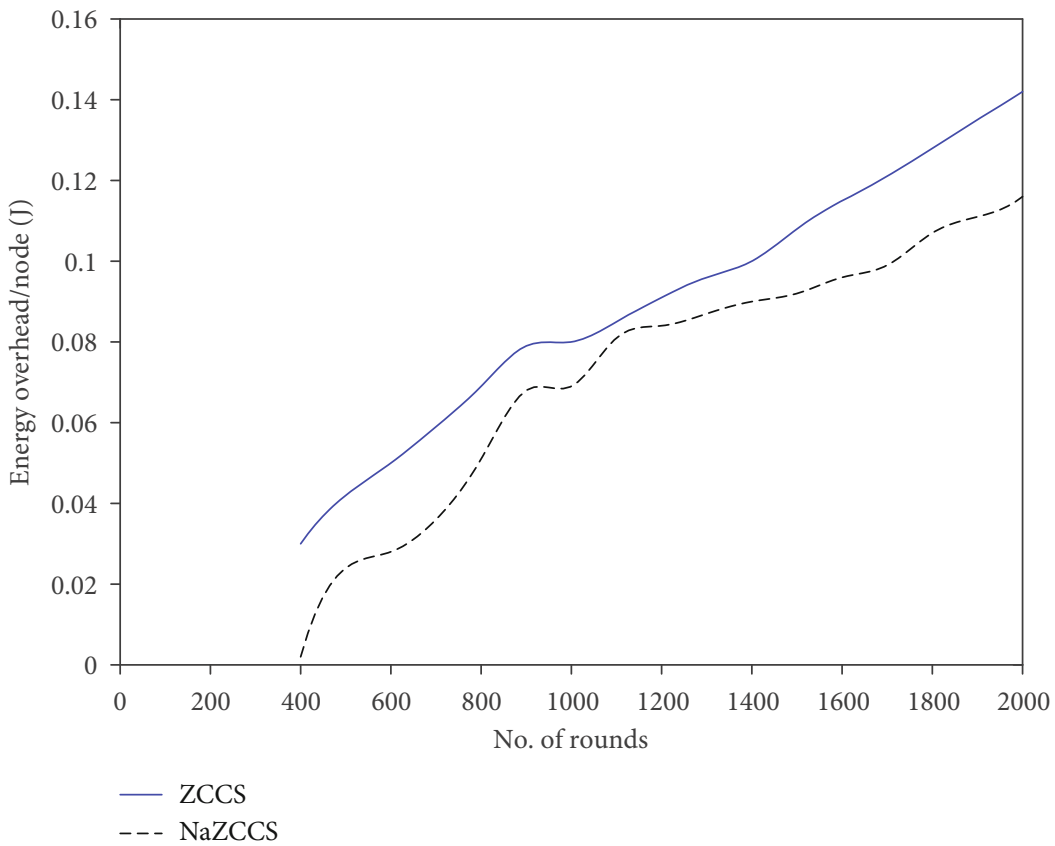

Figure 8: Average energy overhead/round under node density 0.1 .

consideration calculate the node density with the assistance of poissrnd MATLAB function [31]. An average of 5 numbers of runs with 45,80 , and 114 nodes under the area 60 $\times 60$ yields node densities $0.1,0.2$, and 0.3 , respectively, as shown in Figure 2. All results are based on the number of nodes generated by poissrnd function on a run. Wireless sensor networks support hierarchical communication, where sensing and data transmission to a destination are carried in rounds. The number of rounds represents the schedule for the overall sensor network communication.
In the first set of experiments, the number of charging requests successfully fulfilled by the network is considered to assess the charging throughput of ZCCS and NaZCCS under varying node densities. Figure 3 shows a comparison between two schemes at node density 0.1. Figure 4 compares two schemes at node density 0.2 , whereas Figure 5 shows a comparison between two schemes at node density 0.3 . Figure 6 shows a comparison of charging throughput under different node densities for NaZCCS, whereas Figure 7 compares charging throughput under 


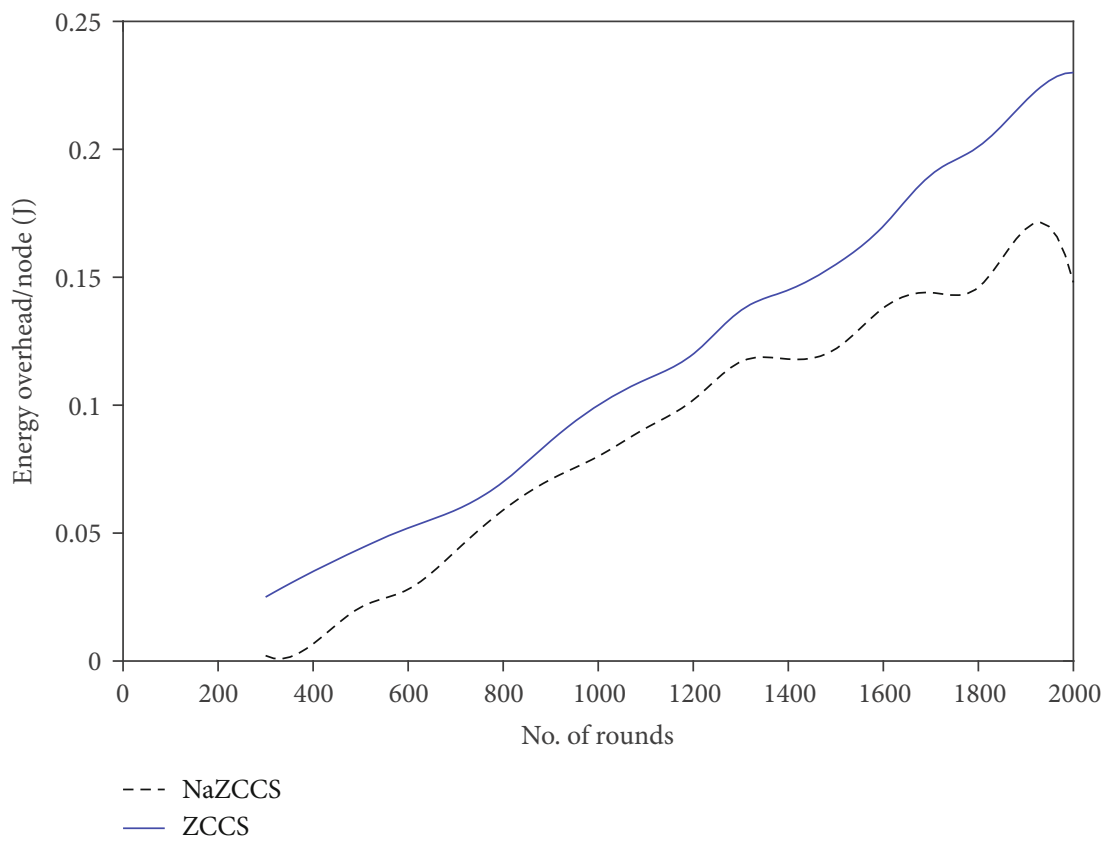

Figure 9: Average energy overhead/round under node density 0.2.

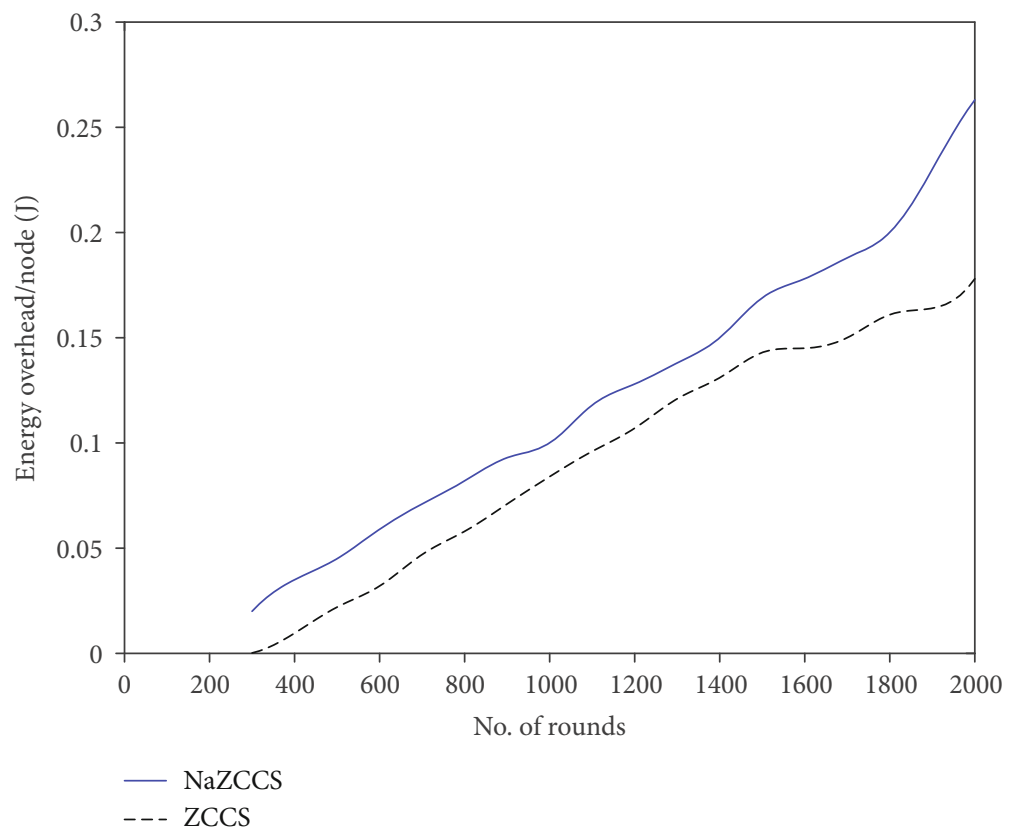

Figure 10: Average energy overhead/round under node density 0.3.

different node densities for ZCCS. From these evaluations, it is analysed that the performance difference between NaZCCS and ZCCS is less under low node density. Both schemes demonstrate similar charging throughput. However, this difference increases with an increase in node densities. It is evident from the results that ZCCS performs better under high densities in terms of charging throughput, as it can serve a greater number of nodes per unit time by exploiting on-demand local charging as compared to NaZCCS. It can also be observed from Figures 6 and 7 that a change in node densities does not significantly affect the charging throughput of NaZCCS; however, in case of ZCCS, it results in significant improvement in charging throughput.

5.2.2. Energy Overhead under Varying Node Densities. Energy overhead incurred by both ZCCS and NaZCCS is evaluated under different node densities and is shown in Figures 812. Figure 8 compares the energy overhead of both schemes for node density 0.1. Figure 9 presents a comparison between two schemes under node density 0.2 . Figure 10 compares them under node density 0.3 . 


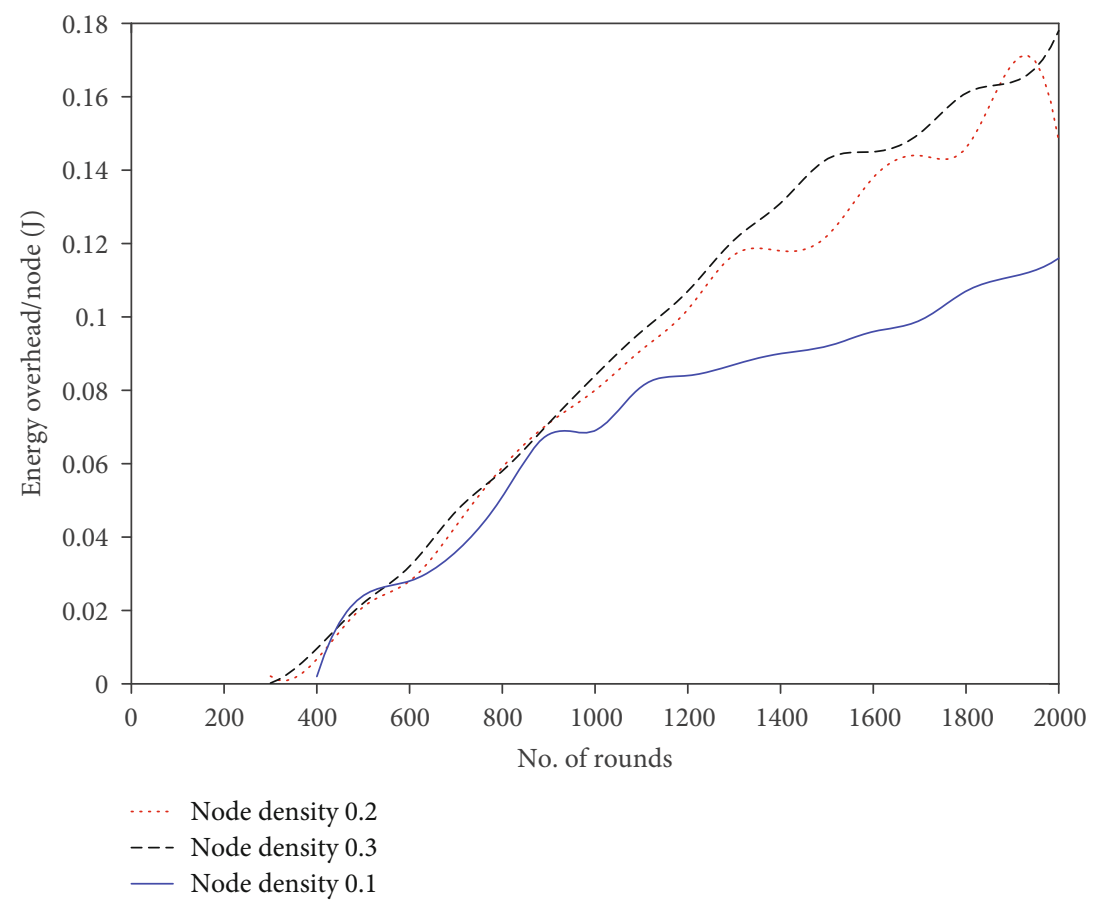

FIGURE 11: Average energy overhead for NaZCCS/round under different node densities.

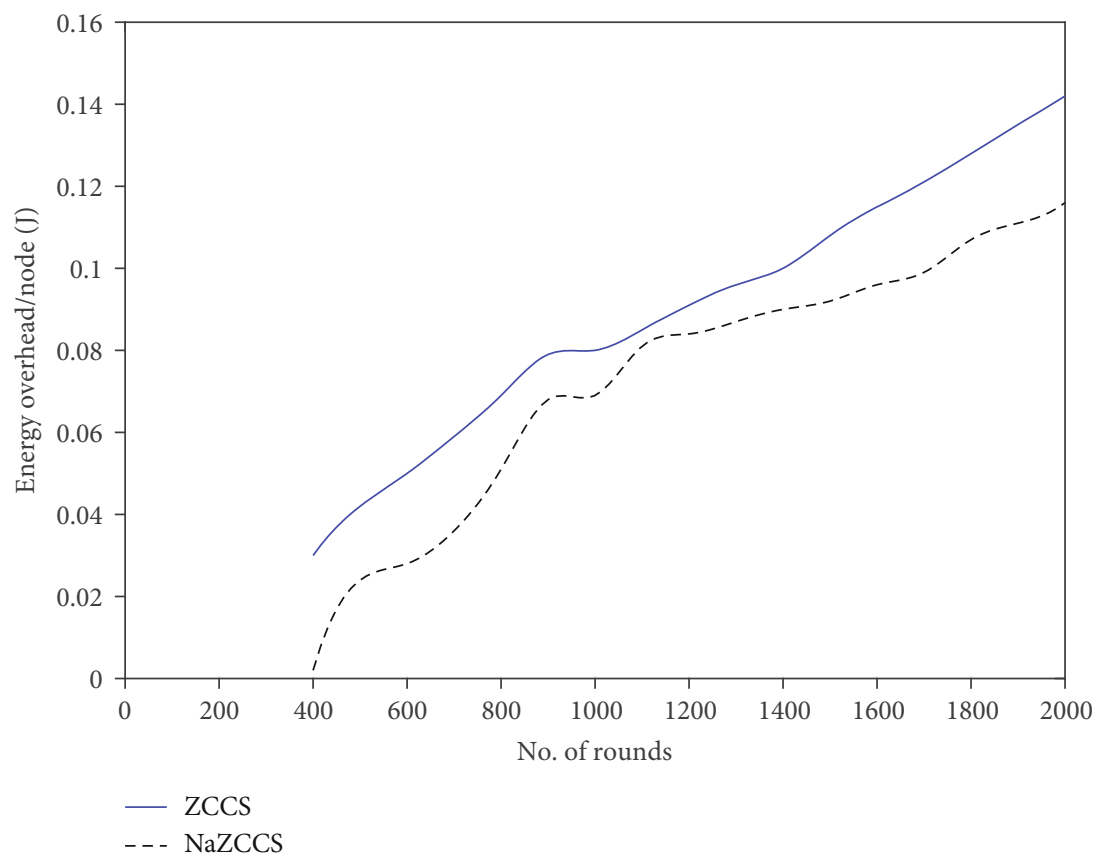

FIGURE 12: Average energy overhead for ZCCS/round under different node densities.

Figure 11 gives a comparison of energy overhead under different node densities of NaZCCS, whereas Figure 12 shows a comparison under different node densities for ZCCS. Under node density 0.1 as shown in Figure 8, both schemes demonstrate similar energy overhead. However, with an increase in node density, energy overhead incurred by ZCCS is higher than that by NaZCCS. This overhead is attributed to control packets exchanged before initiating recharging process.
However, on-demand recharging method of ZCCS compensates this loss through local charging in a timely fashion without any compromise on network lifetime.

5.2.3. Average Charging Latency. Average charging latency reflects charging efficiency of a recharging scheme. A small charging latency indicates a high probability of connectivity with a better network lifetime. For both schemes, average charging latency is evaluated under 100 nodes deployed in 
TABLE 2: Average charging latency.

\begin{tabular}{lc}
\hline Scheme & Average charging latency \\
\hline ZCCS & $27 \mathrm{~s}$ \\
NaZCCS & $47 \mathrm{~s}$ \\
\hline
\end{tabular}

an area of $60 \times 60$ divided into 4 regions each with $15 \times 15$ size for 5 simulation runs. Average charging latency for both algorithms under this deployment is given in Table 2. From the table, it can be observed that the average charging latency observed by the NaZCCS is double the average charging latency of ZCCS. ZCCS demonstrates this excellent charging latency improvement due to its on-demand local charging supported by the local chargers deployed in each region. It balances the load across the local chargers thereby reducing the charging latency. On the other hand, in NaZCCS, all the charging requests are serviced by a single global charger which may queue the requests longer under high traffic loads.

\section{Conclusions}

This work presents an efficient collaborative wireless energy recharging for wireless rechargeable sensor networks. The proposed algorithm exploits the idea of local charger deployment to minimize charging time of a WSN deployment with improved charging throughput. Charging throughput and charging latency are critical for the continuous operation of a WSN deployed in critical regions. Experiment results reveal that ZCCS achieves better charging throughput and charging latency compared to NaZCCS. Our future work will investigate energy overhead incurred by the ZCCS. Further, to overcome the charging overhead, we plan to propose a deep reinforcement learning-enabled power transfer technique.

The technique will formulate recharging policies based on the neural network and active monitoring of wireless sensor network.

\section{Data Availability}

The data used to support the findings of this study are available from the corresponding author upon request.

\section{Conflicts of Interest}

The authors declare that there is no conflict of interest regarding the publication of this paper.

\section{Acknowledgments}

We would like to acknowledge the support from the National Social Science Fund of China (No. 18BGL200), the National Key Statistical Science Research Project (No. 2016LZ11), and the support from the Research on Major Theories and Practices in Shandong Province (No. 18BSJ03). This research work is supported by the SUT research and development fund.

\section{References}

[1] W. Cong, L. Ji, and Y. Yuanyuan, "Wireless rechargeable sensor networks status and future trends," Journal of Communications, vol. 10, no. 9, pp. 696-706, 2015.

[2] A. Kurs, A. Karalis, R. Moffatt, J. D. Joannopoulos, P. Fisher, and M. Soljacic, "Wireless power transfer via strongly coupled magnetic resonances," Science, vol. 317, no. 5834, pp. 83-86, 2007.

[3] H. Dai, Y. Liu, G. Chen, X. Wu, and T. He, "Safe charging for wireless power transfer," in IEEE INFOCOM 2014 - IEEE Conference on Computer Communications, pp. 1105-1113, Toronto, ON, Canada, April 2014.

[4] C. Yang and K. W. Chin, "On nodes placement in energy harvesting wireless sensor networks for coverage and connectivity," IEEE Transactions on Industrial Informatics, vol. 13, no. 1, pp. 27-36, 2017.

[5] R. Deng, S. He, P. Cheng, and Y. Sun, "Towards balanced energy charging and transmission collision in wireless rechargeable sensor networks," Journal of Communications and Networks, vol. 19, no. 4, pp. 341-350, 2017.

[6] Y. Zhang, S. He, and J. Chen, "Data gathering optimization by dynamic sensing and routing in rechargeable sensor networks," IEEE/ACM Transactions on Networking, vol. 24, no. 3, pp. 1632-1646, 2016.

[7] S. Chen, P. Sinha, N. B. Shroff, and C. Joo, "A simple asymptotically optimal joint energy allocation and routing scheme in rechargeable sensor networks," IEEE/ACM Transactions on Networking, vol. 22, no. 4, pp. 1325-1336, 2014.

[8] C. Shao, H. Roh, T. Kim, and W. Lee, "Multisource wireless energy harvesting-based medium access control for rechargeable sensors," IEEE Transactions on Consumer Electronics, vol. 62, no. 2, pp. 119-127, 2016.

[9] M. S. I. Khan, J. Misic, and V. B. Misic, "Zoning and relayingbased mac protocol with RF recharging," IEEE Transactions on Vehicular Technology, vol. 66, no. 2, pp. 1835-1844, 2017.

[10] L. Xie, Y. Shi, Y. T. Hou, W. Lou, H. D. Sherali, and S. F. Midkiff, "Multi-node wireless energy charging in sensor networks," IEEE/ACM Transactions on Networking, vol. 23, no. 2, pp. 437-450, 2015.

[11] S. Zhang, J. Wu, and S. Lu, "Collaborative mobile charging," IEEE Transactions on Computers, vol. 64, no. 3, pp. 654-667, 2015.

[12] A. Madhja, S. Nikoletseas, and T. P. Raptis, "Hierarchical, collaborative wireless charging in sensor networks," in 2015 IEEE Wireless Communications and Networking Conference (WCNC), pp. 1285-1290, New Orleans, LA, USA, March 2015.

[13] L. Xie, Y. Shi, Y. T. Hou et al., "A mobile platform for wireless charging and data collection in sensor networks," IEEE Journal on Selected Areas in Communications, vol. 33, no. 8, pp. 15211533, 2015.

[14] Y. Zhang, S. He, and J. Chen, "Near optimal data gathering in rechargeable sensor networks with a mobile sink," IEEE Transactions on Mobile Computing, vol. 16, no. 6, pp. 1718-1729, 2017.

[15] Y. Shu, K. G. Shin, J. Chen, and Y. Sun, "Joint energy replenishment and operation scheduling in wireless rechargeable sensor networks," IEEE Transactions on Industrial Informatics, vol. 13, no. 1, pp. 125-134, 2017.

[16] T. Liu, B. Wu, H. Wu, and J. Peng, "Low-cost collaborative mobile charging for large-scale wireless sensor networks," 
IEEE Transactions on Mobile Computing, vol. 16, no. 8, pp. 2213-2227, 2017.

[17] X. Rao, P. Yang, Y. Yan, H. Zhou, and X. Wu, "Optimal recharging with practical considerations in wireless rechargeable sensor network," IEEE Access, vol. 5, no. 5, pp. 44014409, 2017.

[18] C. Lin, D. Han, J. Deng, and G. Wu, " $\mathrm{P}^{2} \mathrm{~S}$ : a primary and passer-by scheduling algorithm for on-demand charging architecture in wireless rechargeable sensor networks," IEEE Transactions on Vehicular Technology, vol. 66, no. 9, pp. 8047-8058, 2017.

[19] Y. Shu, H. Yousefi, P. Cheng et al., "Near-optimal velocity control for mobile charging in wireless rechargeable sensor networks," IEEE Transactions on Mobile Computing, vol. 15, no. 7, pp. 1699-1713, 2016.

[20] C. Lin, B. Xue, Z. Wang, D. Han, J. Deng, and G. Wu, "DWDP: a double warning thresholds with double preemptive scheduling scheme for wireless rechargeable sensor networks," in 2015 IEEE 17th International Conference on High Performance Computing and Communications, 2015 IEEE 7th International Symposium on Cyberspace Safety and Security, and 2015 IEEE 12th International Conference on Embedded Software and Systems, pp. 503-508, New York, NY, USA, August 2015.

[21] C. Lin, G. Wu, M. S. Obaidat, and C. W. Yu, "Clustering and splitting charging algorithms for large scaled wireless rechargeable sensor networks," Journal of Systems and Software, vol. 113, pp. 381-394, 2016.

[22] C. Lin, J. Zhou, C. Guo, H. Song, G. Wu, and M. S. Obaidat, "Tsca: a temporal-spatial real-time charging scheduling algorithm for on-demand architecture in wireless rechargeable sensor networks," IEEE Transactions on Mobile Computing, vol. 17, no. 1, pp. 211-224, 2018.

[23] C. Lin, Z. Wang, D. Han, Y. Wu, C. W. Yu, and G. Wu, "TADP: enabling temporal and distantial priority scheduling for on-demand charging architecture in wireless rechargeable sensor networks," Journal of Systems and Software, vol. 70, pp. 26-38, 2016.

[24] T. Zou, W. Xu, W. Liang, J. Peng, Y. Cai, and T. Wang, "Improving charging capacity for wireless sensor networks by deploying one mobile vehicle with multiple removable chargers," Ad Hoc Networks, vol. 63, pp. 79-90, 2017.

[25] L. Fu, L. He, P. Cheng, Y. Gu, J. Pan, and J. Chen, "ESync: energy synchronized mobile charging in rechargeable wireless sensor networks," IEEE Transactions on Vehicular Technology, vol. 65, no. 9, pp. 7415-7431, 2016.

[26] Y. Ma, W. Liang, and W. Xu, "Charging utility maximization in wireless rechargeable sensor networks by charging multiple sensors simultaneously," IEEE/ACM Transactions on Networking, vol. 26, no. 4, pp. 1591-1604, 2018.

[27] A. Ali, Y. Ming, S. Chakraborty, S. Iram, and T. Si, "The augmented approach towards equilibrated nexus era into the wireless rechargeable sensor network," Symmetry, vol. 10, no. 11, p. 639, 2018.

[28] A. Ali, Y. Ming, T. Si, S. Iram, and S. Chakraborty, "Enhancement of RWSN lifetime via firework clustering algorithm validated by ANN," Information, vol. 9, no. 3, p. 60, 2018.

[29] S. Soni and M. Shrivastava, "Novel wireless charging algorithms to charge mobile wireless sensor network by using reinforcement learning," SN Applied Sciences, vol. 1, no. 9, article 1052, 2019.
[30] K. Ota, M. Dong, J. Gui, and A. Liu, "QUOIN: incentive mechanisms for crowd sensing networks," IEEE Network, vol. 32, no. 2, pp. 114-119, 2018.

[31] D. C. Huang and J. H. Lee, "A dynamic n threshold prolong lifetime method for wireless sensor nodes," Mathematical and Computer Modelling, vol. 57, no. 11-12, pp. 2731-2741, 2013. 